Turton et al (2001) describe substantia morbidity in pregnant women whose previous pregnancy (or pregnancies) had ended in spontaneous loss after 18 weeks' gestation. She found that $21 \%$ of women reporting stillbirth as a criterion A stressor had post-traumatic stress disorder (PTSD) symptoms at caseness level in the third trimester of the next pregnancy. Turton et al conclude that women are vulnerable to PTSD in the pregnancy following stillbirth.

I take issue with these findings. There are several methodological problems with the study. First, stillbirth is not defined as pregnancy loss after 18 weeks' gestation. An infant born after the 28th week of gestation who does not breathe after birth or show any other sign of life is termed a stillbirth (Beischer \& Mackay, 1988). Hence, by definition, Turton et al have included 41 women (out of their total number of 66 subjects) who have had miscarriages. It would have been better to report foetal loss figures on babies with a birth-weight of $<500 \mathrm{~g}$, which is current widespread practice. Second, the authors state that 14 out of 66 women did not see their stillborn infants. No reason is given for this. Was this because of the gestational age of the infant ( $<28$ weeks' gestation)? Third, the use of the term PTSD must be questioned. The authors describe stillbirth as a criterion A stressor. One would therefore expect the onset of PTSD within 6 months of the stillbirth. The authors appear to have ignored this time criterion in making a diagnosis of PTSD (World Health Organization, 1993). Similarly, it is difficult to see how the persistent avoidance criterion (criterion C) was met. None of the subjects avoided pregnancy but became pregnant following stillbirth. What the authors describe are symptoms precipitated by the subsequent pregnancy, with the previous 'stillbirth' as a vulnerability or predisposing factor. Perhaps the diagnosis of adjustment disorder would be more appropriate

Beischer, N. A. \& Mackay, E.v. (1988) Obstetrics and the Newborn. London: BaillièreTindall.

Turton, P., Hughes, P., Evans, C. D. H., et al (200I) Incidence, correlates and predictors of post-traumatic stress disorder in the pregnancy after stillbirth. British Journal of Psychiatry, 178, 556-560.

World Health Organization (1993) The ICD-10 Classification of Mental and Behavioural Disorders. Diagnostic Criteria for Research. Geneva: WHO.

J. D. Sheehan University Department of Adult Psychiatry, Mater Misericordiae Hospital, 62-63 Eccles Street, Dublin 7, Republic of Ireland
Author's response: Legal and operational definitions of stillbirth have changed over time, partly in response to medical advances in pre-term baby care which continue to push back the technical age of viability. UK law currently defines stillbirth as the birth of a dead child after 24 weeks' gestation (the legal age of viability). Definitions also vary across nations; in Australia for example, the birth of a dead child after the 20th week of pregnancy is described as stillbirth. Clinicians in every country tend to use parents' experience of their baby's maturity as a guide and generally recognise a loss in the second half of pregnancy as representing a lost child to the parents. As 18 weeks is the gestational age when mothers typically detect foetal movement, and because mothers deliver in the labour ward rather than the gynaecology ward after this date, we operationally defined any infant born without sign of life after 18 weeks' gestation as stillborn.

Women whose pregnancy had reached 28 weeks $(n=25)$ were significantly more likely to have seen their dead baby than those whose pregnancy ended before 28 weeks $(n=41)$. However, as the paper reports, there was no significant association between gestational age and PTSD.

We reported both current and lifetime prevalence rates for PTSD. Lifetime diagnosis rates were higher than for current diagnosis, presumably reflecting Dr Sheehan's point regarding the time criterion. None the less, the high rate of PTSD in the pregnancy following stillbirth compared with 1 year postpartum (birth of healthy baby) does suggest that pregnancy may act as a reactivating stressor, as the paper suggests.

Section C of the PTSD interview identifies seven items associated with 'persistent avoidance'; subjects are required to have above-threshold scores on four or more to qualify. Avoidance of another pregnancy would be a singularly harsh and absolute criterion to apply, as well as being unrelated to the terms of the assessment interview, which shows close correspondence with DSM-III-R standards (American Psychiatric Association, 1987).

\section{American Psychiatric Association (1987)}

Diagnostic and Statistical Manual of Mental Disorders (3rd edn, revised) (DSM-III-R). Washington, DC: APA.

P.Turton University of London Parent Child Research Group, Jenner Wing, St George's Hospital Medical School, London SWI7 ORE, UK

\section{Psychiatric services in developing countries}

We read with interest the editorial on community psychiatry in developing countries (Jacob, 2001). Historically, in the West, community psychiatry arose in the context of the deinstitutionalisation movement and anti-psychiatry. In developing countries, however, the impetus for developing community-based care was the lack of universally accessible services. Thus, without any ideological baggage to contend with, the emphasis should be on integrated services rather than an artificial schism between hospital and community psychiatry.

We agree with Jacob that psychiatrists should concentrate on what they know best - the identification and treatment of mental illness. The mental health programmes in many developing countries set lofty goals of primary prevention that cannot succeed unless backed by overall social and economic development. But we take issue with his inclusion of epilepsy as a potential target of community psychiatry. It is the authors' experience, while working at the Community Psychiatry Unit at Bangalore, India, that this results in the programme becoming a glorified antiepileptic medication clinic.

Jacob's criticism of vertical mental health programmes ignores the practical reality that there is a limit to what generic health workers can deliver given their commitments to other public health programmes such as immunisation. A practical way of getting around this would be to have mental health workers, based at primary health centres, whose skills are intermediate between community psychiatric nurses and generic health workers. There is also a need to develop simple psychosocial interventions which can be delivered by these workers and draw from the strengths of the family or the local community. Community-based rehabilitation is also a priority area as the prevalent concept of good prognosis of mental disorders in developing countries is being challenged (Mojtabai et al, 2001).

One of the stated goals of community psychiatry is to deliver evidence-based treatments to people with mental disorders (Szmukler \& Thornicroft, 2001). It may be heartening for psychiatrists in developing countries to know that the conventional psychotropic medications still remain firstline treatments (Geddes et al, 2001; Barbui $\&$ Hotopf, 2001). The challenge is to ensure 